\title{
Los paradigmas del desarrollo y su evolución: Del enfoque económico al multidisciplinario
}

\section{The paradigms of development and their evolution: From the economic to the multidisciplinary approach}

Carlos Iturralde Durán es investigador de la Universidad Politécnica Salesiana (Ecuador). (carlos.iturralde@hotmail. com) (http://orcid.org/0000-0002-0300-7748)

\begin{abstract}
Resumen
El presente artículo expone la evolución del concepto de desarrollo, cuyo germen antecede al surgimiento de la Economía como ciencia, presentando de forma resumida las teorías que tuvieron mayor reconocimiento por sus contribuciones, revelándose, a través de la investigación bibliográfica, los hitos que marcaron avances significativos en el conocimiento sobre este fenómeno social, hallándose cuatro estadios: el primero que confundió al desarrollo con crecimiento, orientando las políticas al incremento del PIB, seguido de la incorporación de la dimensión social desde el enfoque económico agregando la (re)distribución de la renta como variable, que fue superado con el aporte de otras ciencias como la Sociología, la Política y el Derecho, creando enfoques multidisciplinarios que hoy incluyen a la dimensión ambiental, gestando una nueva concepción cuyo enfoque más aceptado actualmente es el «Desarrollo Humano Sostenible» propuesto por el PNUD, que incorpora el enfoque de capacidades de Sen y principios de sostenibilidad, facilitando el acuerdo plasmado en la Agenda 2030 que planteó diecisiete objetivos desagregados en ciento sesenta y nueve metas en las esferas económica, social y ambiental, siendo tal vez el mayor reto la creación de una cultura ambiental que promueva nuevos valores, y en otros casos retome valores ancestrales, donde la concienciación, producto de un esquema educativo mejorado complementado con un aparataje político ajustado a las demandas socio-ambientales, resultan claves.
\end{abstract}

\begin{abstract}
This article presents the evolution of the concept of development, whose origin comes from the emergence of Economics as a science, presenting concisely the theories with more recognition for their contributions; and revealing, through bibliographic research, the milestones that marked significant advances in the knowledge about this social phenomenon. Four stages were observed: the first that confused development with growth guiding policies to increase GDP; the other is the incorporation of the social dimension from the economic approach by adding the (re) distribution of income as a variable, which was overcome with the contribution of other sciences such as Sociology, Politics and Law, creating multidisciplinary approaches that include the environmental dimension contemplating perspectives of hard sciences such as Physics and Biology, developing a new conception whose approach most accepted now is that of sustainable human development proposed by the UNDP, which incorporates Sen's approach to capabilities and sustainability principles, facilitating the agreement embodied in the 2030 Agenda that set out seventeen disaggregated objectives in one hundred and sixty-nine goals in the economic, social and environmental spheres; this is perhaps the greatest challenge in the creation of an environmental culture that promotes new values, and in other cases, regain ancestral values, where awareness, the product of an improved educational scheme complemented by a political apparatus adjusted to socio-environmental demands are key elements in the process.
\end{abstract}

\section{Palabras clave I keywords}

Teoría del desarrollo, crecimiento económico, capacidades, desarrollo económico y social, desarrollo humano, desarrollo sustentable.

Development theory, economic growth, capabilities, economic and social development, human development, sustainable development.

Cómo citar: Iturralde Durán, C. (2019). Los paradigmas del desarrollo y su evolución: del enfoque económico al multidisciplinario. Retos Revista de Ciencias de la Administración y Economía, 9(17), 7-23. https://doi.org/10.17163/ret.n17.2019.01. 


\section{Introducción}

Los motivos que explican el progreso de unas economías y el rezago de otras, las razones por las que un número significativo de personas vive bajo el umbral de la pobreza, los medios más efectivos para cerrar la brecha entre ricos y pobres, entre otras muchas interrogantes relevantes para la prosperidad del ser humano, requieren de la comprensión de las características y componentes de conceptos como riqueza, desigualdad, pobreza y desarrollo, constituyéndose en el punto de partida para formular teorías, modelos, metodologías y políticas que intenten mejorar la calidad de vida de la población. Estas teorías mantendrán su validez siempre que expliquen la realidad con un grado de acierto aceptable, y hasta que nuevas teorías presenten argumentos de mayor convicción y completitud, dando paso a la acumulación de conocimientos y al avance de la ciencia.

El estudio del desarrollo se formalizó con el surgimiento de la «Teoría del Desarrollo» como rama de la Economía, que inicialmente tomó elementos de la macroeconomía, la microeconomía y la Economía Política para sus análisis, creando un sesgo hacia la dimensión económica que al evidenciarse insuficiente requirió del apoyo de otras ciencias, creando enfoques multidisciplinarios que continúan en evolución, situación que se expone en este artículo sustentado en el análisis bibliográfico que tiene como objetivo presentar de forma resumida las teorías que han tenido mayor reconocimiento por sus aportes a la comprensión del desarrollo, señalando los hitos más importantes que marcaron avances en el conocimiento sobre este fenómeno social.

\section{Revisión teórica}

A lo largo de la historia de la humanidad se encuentran reflexiones sobre la forma de mejorar el nivel de vida y alcanzar la felicidad, entre las cuales destaca el pensamiento aristotélico que asoció la vida digna con la producción y el consumo, incluyendo la importancia del trabajo y el ocio en el bienestar (Martínez-Echevarría \& Crespo, 2011), postura que fue enriquecida con los aportes de la escuela clásica que marcaron el nacimiento de la Economía como ciencia, señalando que la riqueza es la producción, mas no los metales preciosos o el dinero como suponían los mercantilistas, siendo prioritario incrementar la productividad del capital y el trabajo para aumentarla, y con ello, lograr el desarrollo de la nación, proponiendo al libre mercado como el mecanismo más eficiente para este fin.

La hegemonía de la tesis clásica duró hasta 1929 cuando sus teorías y políticas resultaron insuficientes para explicar la «Gran Depresión» y sacar a los países de la crisis, surgiendo la escuela keynesiana que propuso estimular la demanda agregada utilizando políticas fiscales y monetarias para reactivar el PIB y generar empleo, concibiendo al Estado como moderador de los ciclos económicos, pero continuando con la premisa de que el propósito más importante es el crecimiento.

Durante el periodo de postguerra, en el que Estados Unidos se posicionó como potencia dominante e implementó el «Plan Marshall» para reconstruir Europa Occidental, a la vez que robusteció sus relaciones con regiones donde el «fantasma» del comunismo de la extinta Unión Soviética quería renacer, las desigualdades entre países desarrollados y subdesarrollados se amplió, tornándose en tema de investiga- 
ción académica que se abordó desde la «Teoría del Desarrollo» que se consolidó como disciplina económica.

A continuación se presentan las líneas de pensamiento que tuvieron mayor aceptación al momento de su difusión, lo que permite observar la evolución del concepto de desarrollo desde la postura económica hasta la multidisciplinaria.

\subsection{Teoría de la Modernización}

Esta teoría sostiene que para alcanzar el desarrollo hay que superar varias etapas de un camino que ya fue recorrido por las grandes potencias cuyos pasos debían ser imitados por los países subdesarrollados, requiriendo que los valores occidentales reemplacen a los tradicionales, pues erróneamente los asumieron incompatibles. Los modelos relevantes de esta vertiente son los siguientes:

- Modelo de sectores duales de Arthur Lewis: Concibe al desarrollo como el crecimiento per cápita sostenido que transforma una economía tradicional, caracterizada por el estancamiento y la subsistencia, en una economía moderna, centrada en la expansión capitalista, considerando fundamental el incremento de la productividad industrial implementando tecnologías, lo que aumenta la demanda de mano de obra, y con ello, los salarios del sector, motivando la migración campo-ciudad. La escasez de mano de obra en el campo provoca el alza de los salarios en el sector primario presionando a los terratenientes a implementar tecnologías que incrementen la productividad del trabajador agrícola, dejando la economía tradicional e incorporándose a la modernidad (Lewis, 1958).

- Debido a la creencia de que sólo las ganancias de los capitalistas eran capaces de financiar la inversión, pues los ingresos de la clase media y los salarios de subsistencia de la clase baja resultaban insuficientes para alcanzar un nivel de ahorro significativo, señalaron que la importancia residía en el crecimiento y no en la distribución de la renta, pues ésta debía estar sesgada a favor de los burgueses (Gutierrez, 2007).

- Modelo de las etapas del desarrollo de W. Rostow: Asume al desarrollo como el eslabón más alto entre cinco etapas progresivas, las cuales se enuncian a continuación:

i. Sociedad tradicional: La producción es rústica, agrícola y destinada al consumo más que al comercio, siendo una economía de subsistencia con escasa acumulación de capital.

ii. Condiciones previas al despegue: El Estado fomenta la modernidad, facilitando la importación de bienes de capital y creando la infraestructura necesaria.

iii. Despegue: La industria local implementa nuevas tecnologías experimentando un rápido crecimiento. Requiere que la tasa de inversión supere a la de crecimiento demográfico apoyándose en el ahorro externo.

iv. Madurez: Los avances tecnológicos se han aplicado con efectividad, incrementando la productividad del trabajo, los salarios y la renta nacional. Las economías se proyectan hacia el exterior. Tiene una duración estimada de sesenta años. 
v. Alto consumo en masa: La producción ha crecido significativamente y se ha diversificado orientándose al sector servicios. El Estado está en capacidad de aplicar políticas sociales y el país adquiere presencia internacional (Aguilar, 2017).

\subsection{Teoría estructuralista}

Se gestó en América Latina marcando su inicio en la Conferencia de la Habana de 1947 con el discurso de Raúl Prebish, quien se opuso a la idea de que el subdesarrollo es una etapa del desarrollo y cuestionó la efectividad de las políticas neoliberales indicando que los países periféricos se insertan al comercio internacional exportando materias primas baratas hacia los países desarrollados e importando de ellos bienes de capital y de consumo con alto valor agregado, situación que deteriora sus términos de intercambio e impide la implementación eficaz de nuevas tecnologías en sus procesos productivos, quedando rezagados de los beneficios del progreso (Rapoport \& Guiñazú, 2016).

Con un tinte keynesiano impulsado por la Comisión Económica para América Latina y el Caribe (CEPAL), se propuso la industrialización por sustitución de importaciones (ISI) que requería de la participación activa del Estado como planificador y direccionador de las actividades económicas, utilizando exenciones tributarias, subsidios, eliminación de aranceles a bienes de capital, entre otros incentivos para fomentar la inversión en las industrias nacientes, pero también aplicando barreras de entrada a los bienes provenientes del resto del mundo que podían rivalizar en el mercado local con los productos ofertados por la industria nacional (Ariel, 2015).

De esta manera, el desarrollo sería inicialmente endógeno, dependiendo del consumo interno, mientras que la implementación de los progresos tecnológicos aumentaba progresivamente la productividad del sector industrial abaratando sus costos, hasta llegar el momento en que su competitividad se equipararía con la de las industrias de los países desarrollados. Entonces, los productos con valor agregado se exportarían y se eliminarían las barreras a las importaciones, permitiendo que el país se inserte al comercio internacional en condiciones más justas.

Sin embargo, en gran parte de los países latinoamericanos que adoptaron este modelo, las industrias locales no mejoraron su competitividad debido a que fueron creadas por grupos nacionales de poder asociados con transnacionales que aprovecharon la ausencia de competencia y la demanda cautiva para obtener beneficios extraordinarios, requiriendo del «paternalismo de Estado» para su sostenimiento, generando inflación y déficits fiscales y en balanza de pagos que explican el fracaso del modelo (Polo, 2016).

\subsection{Teoría de la dependencia}

Nace en los años cincuenta adquiriendo fuerza en las dos décadas posteriores. Tomando líneas del neomarxismo y del pensamiento weberiano, propugna la idea de que existen grupos de poder nacionales interesados en que América Latina continúe siendo primario-exportadora, sometiendo a los Estados-nación a una relación donde su desarrollo depende del progreso de los países hegemónicos (Vergara \& Ortiz, 2016). 
Los "países periféricos», pese al deterioro de los términos de intercambio, mantuvieron su oferta exportable consiguiendo competitividad a través de la explotación laboral, lo que contrajo la demanda interna, a la vez que importaron bienes con valor agregado y tecnología obsoleta que no les permitió incrementar su productividad a los niveles de los países del «centro», perpetuando los desequilibrios internos y externos, y consolidando un modelo dual donde el desarrollo y el subdesarrollo son caras opuestas de la misma moneda (Gutierrez, 2007).

Esta teoría diferencia el concepto de «crecimiento» del de «desarrollo económico» aduciendo que el primero no es producto de la implementación de nuevas tecnologías por lo que no contribuye a salir de la estructura de dependencia, mientras que el segundo sí. También propuso mantener el «modelo ISI» pero con una política proteccionista moderada aplicada sobre actividades previamente priorizadas (Hunt, 1989).

\subsection{Teoría neoclásica institucional}

La escuela institucionalista fue fundada en Estados Unidos por Thorstein Veblen y John R. Commons, cuyos estudios se fijaron en el rol de las instituciones en el desarrollo, al que consideraron un proceso evolutivo que se apoya en comportamientos asociados a actividades útiles que crecen conforme se implementan los progresos tecnológicos, a los que llamaron «valores universales» que están alineados con la eficiencia y el beneficio económico, encontrando como resistencia a los «valores culturales» que se asocian a la ética, la moral y la opinión de la comunidad, revelándose el carácter dual de las personas quienes son finalmente las que promueven el cambio (Macagnan, 2013).

Esta escuela, a diferencia del pensamiento clásico, cree que los gustos y preferencias varían y que la racionalidad es limitada pues existe un proceso de aprendizaje circunscrito a la cultura, normas e instituciones, lo cual liga la conducta económica al derecho ya que las leyes regulan las transacciones, por lo que el desarrollo debe estudiarse contemplando además de la dimensión económica, la social y la política (Commons, 1931).

\subsection{Teoría neomarxista de los sistemas mundiales}

Immanuel Wallerstein, principal teórico del análisis sistema-mundo, reconoció que estudiar al Estado-nación de forma aislada sin considerar las condiciones globales es insuficiente, sugiriendo una visión holística que incluya la dinámica de los sistemas de comunicación mundial, los sistemas financieros internacionales, la transformación del conocimiento, la evolución del comercio e incluso los vínculos militares, para lo cual la Economía debía apoyarse en la Sociología, adaptándose a la nueva lógica del sistema capitalista que ignoraba las fronteras en búsqueda de ganancias dentro de un mundo cada vez más integrado al mercado global, generando diferentes estadios de desarrollo que permitía categorizar a los países en periféricos, semiperiféricos y del centro, siendo los últimos los mayores beneficiarios del reparto desigual de la riqueza (González, 2004).

\subsection{Modelo neoliberal de apertura y globalización}

En el contexto de la intensificación de la interconexión entre comunidades distantes y diversas que modificaron sus culturas y fortalecieron sus vínculos económicos, 
sociales y políticos producto de los avances en las Tecnologías de la Información y la Comunicación (TIC) durante los años setenta y ochenta, y la crisis de la deuda externa que se fraguó en Latinoamérica, la «Escuela de Chicago» gestó este modelo acusando al proteccionismo como causal de la crisis, priorizando el desmantelamiento del «Estado del Bienestar» a través de la aplicación de paquetes de reformas sugeridas por el Fondo Monetario Internacional, el Banco Mundial y el Departamento del Tesoro de Estados Unidos, a las que John Williamson llamó el «Consenso de Washington», que iniciaba con la firma de una carta de intención donde el gobierno se comprometía a contraer el gasto público, sobre todo el gasto social, a no-intervenir en el mercado monetario para tener un tipo de interés y un tipo de cambio regidos por la competencia, a eliminar barreras arancelarias y no-arancelarias al comercio internacional, a abrirse a los capitales extranjeros, a privatizar las empresas públicas, a respetar los derechos de propiedad, y en general, a desregular los mercados, a cambio de acceder a préstamos otorgados por los organismos multilaterales (Castañeda \& Díaz-Bautista, 2017).

La implementación de estas políticas, llamadas inicialmente «de austeridad»y posteriormente «de ajuste estructural», derivó en el deterioro de la demanda y oferta agregada que redujo la eficiencia e incrementó la desigualdad y la pobreza, afectando negativamente al desarrollo de quienes las adoptaron (Casas, 2017).

\subsection{Teorías de desarrollo sostenible.}

Durante los años cuarenta aparecieron movimientos civiles y estudios académicos que advertían de la posible crisis ambiental consecuencia de los modelos de desarrollo que impulsaban la producción industrial y el consumo en masas sin contemplar la degradación de los ecosistemas y las restricciones que éstos imponen sobre la calidad de vida actual y futura.

A inicio de los setenta, las Naciones Unidas reconocieron al medio ambiente como una dimensión del desarrollo, situación que se complementó con la creación del «Club de Roma» (Gutierrez, 2007), motivando al debate que se cristalizó en diferentes comisiones y reportes, siendo de particular importancia el informe Brundtland de la Comisión Mundial de Medio Ambiente y Desarrollo que cuestionó el modelo basado en el supuesto de una posibilidad ilimitada de crecimiento y elaboró la definición de «Desarrollo Sostenible», entendido como aquel que permite satisfacer las necesidades de la presente generación sin comprometer la capacidad de las generaciones futuras para satisfacer las suyas (CMMAD, 1988), impulsando la difusión de nuevas teorías de desarrollo, entre las cuales destacan las siguientes:

\subsubsection{Teoría del decrecimiento}

En 1972, después de la publicación del informe del «Club de Roma» llamado Los límites del crecimiento cuya principal autora fue Donella Meadows; Herman Daly, influenciado por el pensamiento de John Stuart Mill, propuso la «Teoría del estado estacionario de equilibrio dinámico» donde el sistema, a través del accionar político consciente, se compone de un stock constante de capital y población que se mantienen por los recursos naturales, existiendo desequilibrios cuando se rebasa la restricción presu- 
puestaria natural, limitada por las fuentes solar y mineral, sobreexplotando recursos de escasez absoluta para satisfacer necesidades relativas y triviales (Daly, 1991).

Georgescu-Roegen (1976) objetó la propuesta de Daly (ob. cit) señalando que el estancamiento anula las posibilidades de mejorar la calidad de vida de países pobres además que el crecimiento se mide por el incremento del producto real sin contemplar las contradicciones en la tasa de agotamiento de los recursos naturales logrando solo aletargar su depredación, cuando lo necesario es reconvertirlos (Naredo, 2011), y dado que el hombre es incapaz de crear y destruir materia o energía pudiendo sólo transformarla, y que por la ley de la entropía existe energía que se disipa en cada proceso, lo sustentable sería reducir la extracción indiscriminada para prolongar la existencia de la humanidad (Zaar, 2018).

Latouche (2009), reconociendo al sistema insostenible, propuso realizar un decrecimiento planificado que eliminara las asimetrías existentes que sobrevaloran los flujos monetarios a la vez que subvaloran los costos físicos y humanos, cuantificando los costos de extracción y omitiendo los de reposición de los recursos naturales, apoyados por un marco institucional sesgado hacia el capital que resulta en el detrimento social y ambiental (Naredo, 2010), lo cual tendría como propósito lograr que las personas vivan «dentro de los límites» en el sentido biosférico (Riechmann, 2004), y para ello resultaría prioritario consolidar una nueva «cultura política» (Morin, 2011).

\subsubsection{Teoría del crecimiento a escala humana}

Max-Neef, Elizalde y Hopenhayn (1986) propusieron la «Teoría de necesidades humanas fundamentales» compuesta de tres subsistemas que permiten una comprensión del desarrollo que supera la perspectiva económica, los cuales son:

- Necesidades: que son parte de la interioridad humana, y por ende inmutables, existiendo nueve de ellos de igual importancia: subsistencia, protección, afecto, entendimiento, creación, participación, ocio, identidad y libertad. La carencia de una necesidad por debajo de un umbral mínimo presistémico provoca pobreza.

- Satisfactores: que son el puente que una las necesidades con los bienes, y son afectados por la cultura y el contexto histórico.

- Bienes: que son materiales y por ende limitados a la biósfera. Su uso potencia los satisfactores atendiendo necesidades.

Entonces, mientras las necesidades y bienes son finitos, los satisfactores son ilimitados, y para alcanzar una sociedad sustentable hay que incrementar la conciencia de la población transformando la cultura hacia una visión ecológica, donde los artefactos estén al servicio de la vida, y no al revés (Elizalde, 2000).

\subsubsection{El desarrollo humano sostenible del PNUD}

El Programa de las Naciones Unidas para el Desarrollo (PNUD) formuló una propuesta multidimensional para abordar el concepto de desarrollo que contempla al sistema humano a partir de la dimensión económica y social, y al sistema natural desde la dimensión ambiental; pretendiendo que el crecimiento económico se logre simultá- 
neamente con la erradicación de la pobreza, el fomento de la equidad, el incremento de las capacidades y libertades humanas, sin vulnerar el medio ambiente, para garantizar la extensión de la existencia de la humanidad.

Mientras que las teorías predecesoras se centraron en el fomento a la producción a partir de la productividad, y en el mejor de los casos consideraron también su distribución, el desarrollo humano sustentable adoptó el enfoque de Amartya Sen, quien propuso como medida las capacidades que tienen las personas para lograr funcionamientos valiosos que les den la libertad de escoger el nivel de vida que tienen motivos para valorar, de tal manera que existe una correlación positiva entre las capacidades, que se traducen en libertades, y el desarrollo humano (Iturralde, 2018).

Así, el núcleo del análisis se trasladó de las actividades económicas a las capacidades de las personas entendidas como el conjunto de funcionamientos básicos y especializados, físicos, legales e intelectuales que poseen para lograr su bienestar. Entonces, la cantidad de activos determinan el bienestar potencial en la medida en que incrementan las capacidades, lo que depende del estado del individuo, pues dos sujetos pueden tener los mismos recursos, pero su disfrute puede diferir según su condición (Urquijo, 2014).

En este sentido, el acceso a recursos monetarios es importante para el desarrollo, pero no es la única arista de interés, pues el propósito es crear un entorno de oportunidades alcanzables que mejoren el nivel vida de la población, para lo cual necesariamente debe incluirse la «dimensión social» que comprende elementos como: salud, educación, ocio y esparcimiento, dignidad, derechos políticos, participación comunitaria, entre otros (Sen, 2000).

Al colocar al ser humano como el fin último del desarrollo, surgió la necesidad de incorporar la dimensión ambiental al concepto, encontrándose los primeros debates sobre la importancia del patrimonio ecológico en la Conferencia de Estocolmo sobre el Medio Humano en 1972 promovida por el PNUD, que posteriormente continuó en la Conferencia de Río sobre Medio Ambiente y Desarrollo en 1992, entre otras importantes intervenciones y convenios que robustecieron el concepto de desarrollo sustentable que hoy es ampliamente aceptado por la comunidad científica.

El PNUD también formuló un nuevo instrumento que se ajusta de mejor manera a la visión del Desarrollo Humano Sustentable, denominado el «Índice de Desarrollo Humano» (IDH) que resulta de la media aritmética de tres dimensiones (ver tabla 1).

Tabla 1. Componentes y variables de medición del IDH

\begin{tabular}{|l|l|}
\hline \multicolumn{1}{|c|}{ Componente } & \multicolumn{1}{c|}{ Variable de medición } \\
\hline Riqueza & PIB per cápita \\
\hline \multirow{3}{*}{ Educación } & $\begin{array}{l}\text { Escolaridad media y esperada } \\
\text { Tasa de alfabetización de adultos } \\
\text { Tasa bruta de matriculación }\end{array}$ \\
\hline Salud & Esperanza de vida al nacer \\
\hline
\end{tabular}

Fuente: PNUD, 2016 
De acuerdo a la puntuación obtenida, el IDH categoriza a los países de la siguiente manera:

- Desarrollo muy alto: 0,80 o más puntos

- Desarrollo alto: entre 0,70 y 0,79 puntos

- Desarrollo medio: entre 0,55 y 0,69 puntos

- Desarrollo bajo: 0,54 puntos o menos

A partir de este enfoque, en la Conferencia de las Naciones Unidas sobre el Desarrollo Sostenible celebrada en Río de Janeiro en 2012, se plantearon diecisiete «Objetivos de Desarrollo Sustentable» (ODS) que direccionan el accionar de los países participantes hacia la erradicación de la pobreza, el cuidado del planeta y la creación de un entorno de paz y prosperidad, sustituyendo a los «Objetivos de Desarrollo del Milenio»(ODM), y coincidiendo con el Acuerdo de París, aprobado en la Conferencia sobre el Cambio Climático (PNUD, 2016).

\section{Hacia una comprensión integradora de los paradigmas}

Hasta el periodo de postguerra donde se constituyó la «Teoría del Desarrollo» como disciplina de la Economía, siendo una de sus precursoras la teoría de la modernización que estudió los pasos que permitieron desarrollarse a las potencias con el afán de que los países con menor desarrollo las imiten, se asumió al crecimiento como sinónimo de desarrollo, direccionando el debate hacia la forma de producir más, utilizando como indicador clave al PIB per cápita, que si bien es relevante, sufre de diversas deficiencias entre ellas: el ser un promedio que no presenta datos sobre la dispersión ocultando información sobre la distribución de la riqueza, además que su cálculo omite datos de la economía sumergida (ilegal e informal) y de actividades que poseen valor pero no son remunerados como el trabajo doméstico, el voluntariado, entre otras., a lo que se debe añadir que no refleja ni la calidad de los bienes ni las externalidades negativas que ocasiona su producción y consumo. Por ejemplo, el PIB de Brasil crecería si se deforesta la amazonía pero se reduciría la calidad de un sinnúmero de servicios ambientales que son determinantes del bienestar, embargando el progreso futuro y desmejorando las condiciones de desarrollo.

Con la teoría de la dependencia, que se nutrió de elementos de la teoría estructuralista, apareció la primera distinción clara entre crecimiento y desarrollo, señalando que la aplicación de tecnologías que ocasiona cambios en la estructura productiva deriva en desarrollo, de lo contrario si la economía se expande sin cambios estructurales sólo hay crecimiento, prevaleciendo la idea neoclásica schumpeteriana de analizar la problemática exclusivamente desde la dimensión económica (Jahan, Mahmud, \& Papageorgiou, 2014).

Aunque los intentos de aumentar el bienestar, medido por el incremento del producto y el consumo en masas, desembocaron en problemas de creciente desigualdad y exclusión social que fueron señalados por los cepalinos dependentistas y estructuralistas, no fue hasta que se propagó la teoría institucional que se superó la perspectiva unidimensional económica agregando la dimensión social, pues se destacó la importancia de los comportamientos humanos para comprender el desarrollo, 
requiriendo la contribución de otras ciencias. Las teorías de los sistemas mundiales y de la globalización, además de aceptar las dimensiones económica y social del desarrollo, ampliaron el alcance que se restringía al Estado-nación contemplando los efectos del contexto global y sus subsistemas.

A partir de ello, se concibe al desarrollo como la capacidad de los países para crear riqueza y promover el bienestar social en el marco de la globalización, reconociendo que la acumulación cuantitativa de capital y la medición del desarrollo con una única variable (el PIB) y una sola dimensión (la económica), eran insuficientes, por lo que debían añadirse los saltos cualitativos que el progreso provoca en la sociedad (Chirinos, Meriño, Martínez, \& Pérez, 2018), no obstante aún no se incorporaba el sistema ambiental, como se observa en la figura 1.

\section{Figura 1. Desarrollo económico y social}

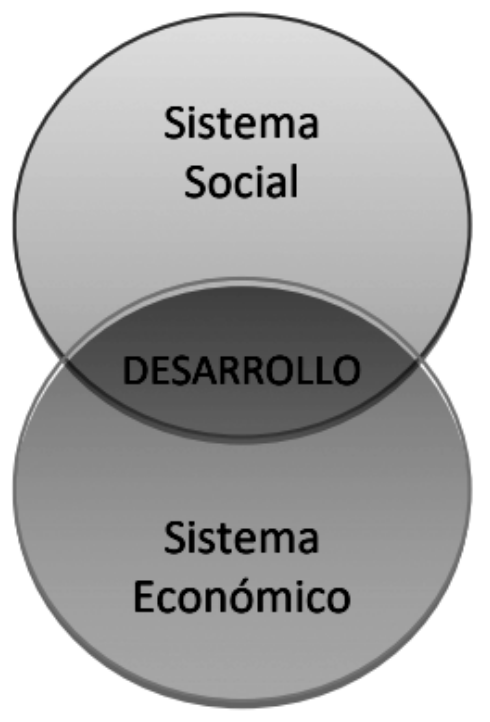

La exclusión del sistema ambiental implica el supuesto que el crecimiento puede ser ilimitado, representando a la curva de oferta con una pendiente positiva que crece infinitamente conforme aumentan los precios, restringiéndose básicamente por la cantidad y productividad de la mano de obra y el capital que provocan una frontera de posibilidades de producción que puede ampliarse con el crecimiento demográfico y los progresos tecnológicos, centrando la discusión en los mecanismos más efectivos para incrementar la productividad marginal de los factores así como la forma más eficiente para que la riqueza se distribuya equitativamente.

Esta lógica capitalista impulsó el consumismo e individualismo, instaurando modelos que pretenden el crecimiento exponencial ignorando la «ley de la entropía» (Rifkin, 2014), desmejorando el capital natural y permitiendo un nivel de vida presente a costas del bienestar de las futuras generaciones (Sempere \& Tello, 2007), pues suponer al mercado como el mecanismo idóneo para determinar la producción 
y distribución, es desconocer el funcionamiento de los sistemas sociales y naturales cuya interrelación desemboca en cambios que pueden tener magnitudes inesperadas en diferentes tiempos que, al deteriorar rápida y significativamente el entorno del ser humano, pone en riesgo la prolongación de su existencia (Bermejo, 2008), tal y como lo advierten diversos reportes, entre ellos, el Informe Planeta Vivo de 2016 que reveló que al 2012 los recursos naturales y servicios ambientales consumidos por los humanos demandó la biocapacidad de 1,6 planetas, por lo que su suministro se realizó extrayendo recursos y devolviendo residuos a una tasa superior a la de su regeneración, además que la población de animales vertebrados se contrajo en $58 \%$ entre 1970 y el año ut supra referenciado (WWF, 2016).

Superar el capitalismo depredador es imperante, y según Gorz (2008), ocurrirá de forma incivilizada a través de catástrofes o por medio de programas basados en nuevos modelos sostenibles, situación que para Martínez Alier (2008) ha motivado a iniciar un sutil proceso de decrecimiento, más que del PIB, del uso de recursos y emisiones, el cual debe intensificarse con el empleo de energías renovables, la construcción de un marco que robustezca conceptos verdes, y la transferencia de nuevos conocimientos que empoderen a la ciudadanía de estos procesos (Barcena, 2011).

La propuesta con mayor aceptación es el «desarrollo humano sustentable» del PNUD que presenta dos avances fundamentales que marcan el concepto de desarrollo:

- El robustecimiento de la dimensión social utilizando el enfoque de capacidades de Sen en lugar de centrarse en la (re)distribución de la riqueza, modificando la perspectiva de equidad y justicia social. Tradicionalmente se asumía que dos agentes con iguales ingresos tienen el mismo bienestar lo cual es falso si, por ejemplo, uno de ellos padece ceguera. De igual manera ocurre si se mide por los activos, pues una mujer por cuestiones religiosas y/o culturales se le impide estudiar o sufragar, aunque tenga acceso a vestido, vivienda o joyas, por lo que teniendo satisfechas sus necesidades motrices y de salud, no lo están sus necesidades políticas, de vida comunitaria y de autorrealización, pues carece de igualdad de derechos impidiéndole desarrollarse apropiadamente.

- La incorporación explícita de la dimensión ambiental, reconociendo que los recursos no renovables son finitos y los renovables tienen un ciclo de producción y reproducción que no necesariamente se corresponde con demanda del mercado, derivando en su sobreexplotación para finalmente devolver a un sistema natural deteriorado los residuos de los procesos de producción y consumo; y aunque existe la hipótesis de la curva ambiental de Kuznets que indica que el daño ambiental crece conforme el país se desarrolla hasta llegar un punto en que la situación se torna en una relación inversa donde un mayor grado de desarrollo se traduce en menor volumen de emisiones, la evidencia empírica es bastante pobre, e incluso contradictoria, por lo que no se la puede asumir como tautológica (Suárez, 2011; Mendaza, 2015; Falconí, Burbano, \& Cango, 2016).

De esta manera se acepta la interconexión orgánica y multidisciplinaria que reconoce la importancia actual y futura del bienestar que fue plasmada en 
la Comisión de Brundtland en 1991 (Carro-Suárez, Sarmiento, \& Rosano, 2017), generando un modelo que abarca por lo menos las tres dimensiones presentadas en la figura 2, donde el punto A simboliza las propuestas de desarrollo que pretenden crecimiento y bienestar social pero depredan los ecosistemas, el modelo de desarrollo B que procura el beneficio social y el cuidado ambiental pero no es sostenible por carecer de la perspectiva económica, el modelo $\mathrm{C}$ donde se cuida el ambiente pero los beneficios del progreso económico se concentran en pocas manos pues no propende a la equidad ni a la justicia social, y el punto D donde convergen las tres dimensiones, que es el desarrollo sustentable entendido como "el diseño de sistema humanos e industriales que aseguren que el uso que hace la humanidad de los recursos naturales no disminuyan la calidad de vida por el impacto en las condiciones sociales la salud humana y el medio ambiente" (Mihelcic \& Zimmerman, 2012, p. 4).

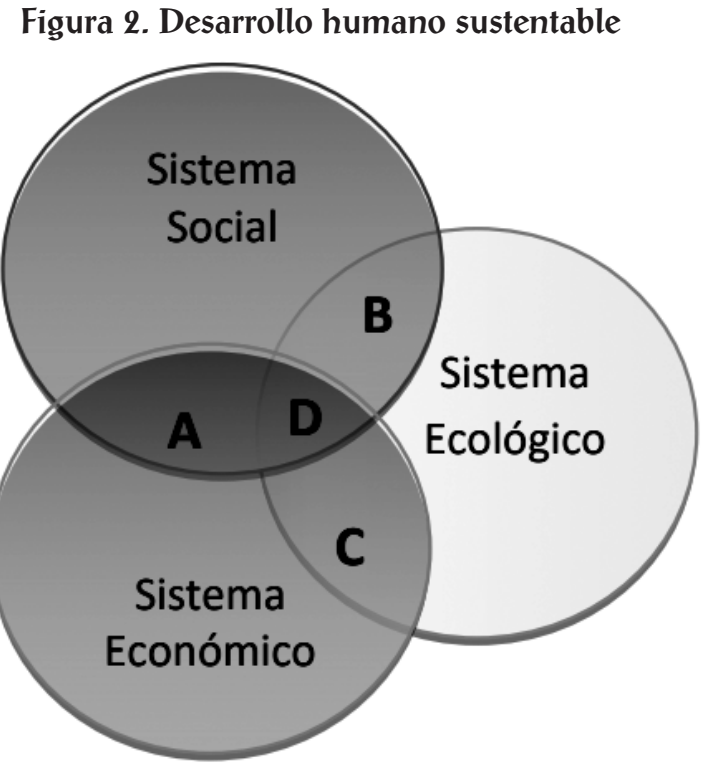

Fuente: Elaboración propia a partir de Salcedo, Rebolloso y Barber (2010, p. 26)

Cada dimensión tiene metas cuya agregación resulta en un nivel de desarrollo humano sustentable, siendo el IDH un esfuerzo por medir el resultado global del modelo. No obstante existen diferentes variables de interés que se estudian con indicadores específicos para cada dimensión, como se ejemplifica en la tabla 2, creando un sistema interconectado donde la variación de un componente afecta a otros, como podría ser el caso de un incremento en los años de escolaridad (de la dimensión social) que deriva en el aumento de la producción (de la dimensión económica) y en mayor conciencia ambiental (de la dimensión ambiental), o el deterioro de la calidad del agua que impacta negativamente sobre la morbilidad infantil y sobre la productividad de un territorio. 
Tabla 2. Ejemplo de variables e indicadores por dimensión de desarrollo

\begin{tabular}{|c|c|c|}
\hline Dimensión & Variables & Indicadores \\
\hline Económica & $\begin{array}{l}\text { Crecimiento económico } \\
\text { Desocupación } \\
\text { Inflación } \\
\text { Balanza de pagos }\end{array}$ & $\begin{array}{l}\text { - Tasa de variación del PIB real per } \\
\text { cápita. } \\
\text { - Tasas de desocupación, subocupa- } \\
\text { ción y ocupación plena. } \\
\text { - Índices de precios al consumidor y } \\
\text { al productor. } \\
\text { - Saldos en balanza de pagos y balan- } \\
\text { za comercial. }\end{array}$ \\
\hline Social & $\begin{array}{l}\text { Educación } \\
\text { Salud } \\
\text { Pobreza } \\
\text { Desigualdad }\end{array}$ & $\begin{array}{l}\text { - Años de escolaridad, tasa de } \\
\text { analfabetismo. } \\
\text { - Tasas de mortalidad y desnutrición. } \\
\text { - Línea de la pobreza, brecha de la } \\
\text { pobreza por consumo, pobreza por } \\
\text { NBI. } \\
\text { - Coeficiente de Gini, distribución de } \\
\text { ingresos por deciles. }\end{array}$ \\
\hline Ecológica & $\begin{array}{l}\text { Calidad del aire } \\
\text { Emisión a la atmósfera } \\
\text { Calidad del agua } \\
\text { Calidad del suelo } \\
\text { Naturaleza } \\
\text { Economía verde }\end{array}$ & $\begin{array}{l}\text { - Concentración media anual de NO2 } \\
\text { y O3. } \\
\text { - Emisiones de gases de efecto inver- } \\
\text { nadero, emisiones de partículas. } \\
\text { - Cantidad de agua utilizada por día, } \\
\text { nivel de agua subterránea, depura- } \\
\text { ción de aguas residuales. } \\
\text { - Pérdida de suelo por erosión. } \\
\text { - Concentración de contaminantes } \\
\text { en los tejidos de organismos vivos, } \\
\text { diversidad de especies silvestres } \\
\text { terrestres. } \\
\text { - Intensidad energética de la econo- } \\
\text { mía, huella ecológica, consumo na- } \\
\text { cional de materiales. }\end{array}$ \\
\hline
\end{tabular}

Fuente: Elaboración propia a partir de SICES (2018); MAPAMA (2016) y BCE (2018)

Se destaca que las diferentes vertientes de pensamiento coinciden que el accionar de las empresas y consumidores es clave, pues sus interacciones afectan al nivel de desarrollo; motivo por el cual, en el marco de la sustentabilidad, se debe superar el comportamiento economicista incorporando la dimensión ambiental en los mode- 
los de negocios y en los hábitos de consumo, lo que requiere del apoyo normativo e institucional del Estado (Moreno, 2017).

\section{Reflexiones finales}

El «desarrollo humano sostenible» abarca el enfoque de capacidades de Sen y la sostenibilidad descrita en el informe Brundtland cuya agregación e interacción proporcionan una visión integral del fenómeno a partir de tres dimensiones: económica, social y ambiental; constituyéndose en la tesis dominante en la actualidad que abarca la temática con una perspectiva multidisciplinaria que considera, además de la Economía, a otras ciencias como la Sociología, la Biología y la Política.

El concepto abstracto de desarrollo que rige hoy responde a una evolución del pensamiento que presenta los siguientes hitos:

- El desarrollo fue tratado como sinónimo de crecimiento económico y estudiado solamente desde la dimensión económica considerando al PIB como indicador clave. Posteriormente, Sen reveló que existen países productores de alimentos que sufren de hambrunas, y que, al incrementar su producción, ésta se exporta hacia países con mayor poder adquisitivo impidiendo superar la crisis alimentaria local, evidenciando que la producción sin distribución no necesariamente produce desarrollo.

- A la «dimensión económica», aun dominante, se le sumó la «dimensión social», contemplando tanto la producción como distribución de la riqueza y su impacto en el bienestar de la población que se medía a través de la utilidad, entendida como la satisfacción al consumir, y la distribución de la renta que debía regirse por mejoras de Pareto, es decir, utilizando políticas que incrementen la posición de agentes menos favorecidos sin reducir la del resto de agentes (Pindyck \& Rubinfeld, 2018).

- Se consolidó la «dimensión social» cambiando el enfoque monetario por el de capacidades de Sen, que recogiendo los conceptos de equidad y justicia social, centró la atención en la generación de oportunidades y en la capacidad para aprovecharlas, requiriendo la creación y fortalecimiento de funcionamientos que les permitan a las personas lograr el nivel de vida que valoran.

- A las dimensiones «económica» y «social»se sumó la «dimensión ambiental», al añadir de manera explícita la relevancia del medio ambiente para el desarrollo, incorporando el derecho de las futuras generaciones a satisfacer sus necesidades, asegurando la conservación y extensión de la vida humana, y consolidando la perspectiva multidimensional que requiere del apoyo de ciencias sociales y naturales, superando definitivamente la visión economicista.

Este concepto de desarrollo centrado en las personas con una perspectiva intergeneracional, en lugar de las instituciones o el mercado, constituye un avance teórico significativo que incorporó el enfoque de Sen advirtiendo que la libertad para alcanzar el nivel de vida valorado por los agentes está en función de sus capacidades y de las oportunidades reales, siendo los objetos-valor el "conjunto de bienes materiales e inmateriales que se someten a un proceso de evaluación para priorizarlos en 
términos de utilidad individual, esto es, placer, felicidad o satisfacción de deseos" (Arteaga \& Solís, 2005, p. 39).

$\mathrm{Al}$ «rol de agencia», entendido como aquello que las personas son libres de hacer y alcanzar, así como las responsabilidades de sus acciones y omisiones que están sujetan a los valores, debe incorporarse el papel que juega la cohesión social pues puede multiplicar el esfuerzo individual además de dirigir su accionar a través de la institucionalidad del estado o del comportamiento comunal.

Entonces, el ejercicio de la libertad individual se enmarca en un entorno social dinámico e interconectado globalmente que provee información en forma de cultura y valores, afectando la conducta de los agentes, quienes bajo la lógica consumista del capitalismo imperante, pretenden maximizar beneficios y gastos suscritos a una ideología que premia el egoísmo y la eficiencia económica, impulsándolos a mantener una postura insostenible que embarga la calidad de vida futura a costa de un beneficio actual superfluo, a lo que Max-Neef et al. (1986) denominaron una «estúpida manera de vivir», ya que se desarrollan capacidades cuya incorrecta aplicación deteriora funcionamientos básicos, limitando sus libertades futuras y atentando contra su propia calidad de vida al punto que "gran parte del esfuerzo científico y tecnológico está directa o indirectamente dirigido hacia asegurar las posibilidades de destruir a toda la especie humana" (Max-Neef, Elizalde, \& Hopenhayn, 1986, p. 145).

Aunque la «cultura ambiental» discursivamente es aceptada, fácticamente es poco aplicada haciendo necesario cultivar nuevos valores, y en otros casos retomar valores ancestrales, proveyendo de información que conciencie sobre la realidad social y ambiental latente, permitiendo reordenar los objetos-valor en una jerarquía que promueva la solidaridad y cuidado ambiental, siendo la educación el derecho y vehículo transcendental del proceso que requiere el acompañamiento de una nueva visión política, así como el esfuerzo de la academia para culminar la construcción del concepto de desarrollo que presenta vacíos teóricos e instrumentales, que aunque han sido mejorados, no logran sintetizar el complejo tema del desarrollo y su interacción entre dimensiones que contempla variables cuantitativas pero también cualitativas de gran subjetividad como podría ser el concepto y nivel de felicidad.

Calificar la convivencia equilibrada del hombre con la naturaleza como una utopía, como un sueño inalcanzable por el que vale la pena luchar, es menospreciar la capacidad adaptativa y la racionalidad de la humanidad sentenciándola a su extinción. Cuando la conciencia social y ambiental se cristalice y el camino que se debe recorrer se clarifique, nuestra especie sabrá, querrá y podrá convivir armónicamente con el medio ambiente, consolidándose una cultura ambiental que regirá sobre el accionar cotidiano, individual y social, y sobre todas las dimensiones y variables del desarrollo.

\section{Referencias}

Aguilar, I. (2017). Principios de desarrollo económico. Tercera edición. Bogotá: EcoEdiciones.

Ariel, F. (2015). Análisis del modelo de industrialización por sustitución de importaciones en América Latina y en Argentina. Una mirada hacia la realidad industrial actual de Argentina. Geografía Digital, 12(24), 1-17. https://dx.doi.org/10.30972/geo.12242164

Arteaga, C., \& Solís, S. (2005). Necesidades sociales y desarrollo humano: un acercamiento metodológico. Ciudad de México: Plaza y Valdés S.A. 
Barcena, I. (2011). ¿Decrecimiento? ¿Si, gracias! 6 tesis a favor de decrecimiento sostenible. Viento Sur, 20(118), 46-54.

BCE. (2018). Información Estadística Mensual Nº 1998. Obtenido de Estadísticas del Banco Central del Ecuador: https://goo.gl/GRLxZ5

Bermejo, R. (2008). Un futuro sin petróleo. Colapsos y transformaciones socioeconómicas. Madrid: Los Libros de Catarata.

Carro-Suárez, J., Sarmiento, S., \& Rosano, G. (2017). La cultura organizacional y su influencia en la sustentabilidad. Revista Estudios Gerenciales, 33(145), 352-365. https://doi.org/10.1016/j. estger.2017.11.006

Casas, J. (2017). Implicaciones de los acuerdos del Fondo Monetario Internacional sobre la pobreza en Colombia. Económicas CUC, 38(1), 9-36. http://dx.doi.org/10.17981/econcuc.38.1.01

Castañeda, V., \& Díaz-Bautista, O. (2017). El Consenso de Washington: algunas implicaciones para América Latina. Apuntes del CENES, 36(63), 15-41. https://doi.org/10.19053/01203053. v36.n63.2017.4425

Chirinos, Y., Meriño, V., Martínez, C., \& Pérez, C. (2018). Emprendimiento sostenible para el desarrollo económico de las PYMES. Revista Espacios, 39(7), 3-14.

CMMAD. (1988). Nuestro Futuro Común. Madrid: Alianza Editorial.

Commons, J. (1931). Institucional economics. American Economic Review, 21(4), 648-657. https://doi. org/10.3917/cep.040.0287

Daly, H. (1991). Steady-state economics. Washington: Island Press.

Elizalde, A. (2000). Desarrollo a Escala Humana: conceptos y experiencias. Interacoes. Revista Internacional de Desenvolvimento Local, 1(1), 51-62.

Falconí, F., Burbano, R., \& Cango, P. (2016). La discutible curva de Kuznet. Obtenido de Biblioteca digital de Flacso Andes: goo.gl/JJc9fL

Georgescu-Roegen, N. (1976). Energy and economic myths. Institutional and analytical economic essays. New York: Pergamon Press Inc.

González, M. (2004). Immanuel Wallerstein, el análisis de los sistemas mundiales y los desafíos a las ciencias sociales. VI Jornada de Sociología, Facultad de Ciencias Sociales de la Universidad de Buenos Aires: https://goo.gl/uoX6WN

Gorz, A. (2008). La salida del capitalismo ya ha empezado. Ecorev, (28), s/p: http://ecorev.org/spip. php?article 640

Gutiérrez, E. (2007). De las teorías del desarrollo al desarrollo sustentable. Historia de la construcción de un enfoque multidisciplinario. Trayectorias, 9(25), 45-60.

Hunt, D. (1989). Economic Theories of Development. An Analysis of Competing Paradigms. London: Harvester Wheatsheaf.

Iturralde, C. A. (2018). La Educación Superior en las cárceles. Los primeros pasos de Ecuador. Alteridad, 13(1), 84-95. https://doi.org/10.17163/alt.v13n1.2018.06

Jahan, S., Mahmud, A. S., \& Papageorgiou, C. (2014). ¿Qué es la economía keynesiana? Obtenido de Finanzas y Desarrollo del FMI: goo.gl/fcR3Bp

Latouche, S. (2009). Pequeño tratado del decrecimiento sereno. Barcelona: Icaria.

Lewis, A. (1958). Teoría del desarrollo económico. Ciudad de México: Fondo de Cultura Económica.

Macagnan, C. (2013). Teoría institucional: Escrito teórico sobre los protagonistas de la escuela institucionalista de economía. BASE - Revista de Administração e Contabilidade da Unisinos, 10(2), 131-141. https://doi.org/10.0.15.173/base.2013.102.03

MAPAMA. (2016). Perfil ambiental de España 2016. Obtenido de Ministerio de Agricultura y Pesca, Alimentación y Medio Ambiente. Calidad y Evaluación Ambiental: goo.gl/sBsHLL

Martínez Alier, J. (2008). El ecologismo igualitarista enraizará sobre todo entre los desposeídos del mundo. Revista Papeles: Papeles de relaciones ecosociales y cambio global, (104), 155-162.

Martínez-Echevarría, M., \& Crespo, R. (2011). Aristóteles y el pensamiento económico: una introducción. Revista Empresa y Humanismo, 14(2), 5-9. 
Max-Neef, M., Elizalde, A., \& Hopenhayn, M. (1986). Desarrollo a escala humana. Una opción para el futuro. Santiago de Chile: CEPAUR.

Mendaza, M. (2015). La curva medioambiental de Kuznets: ¿Un modelo fiable sobre la degradación ambiental? Trabajo Fin de Grado de la Universidad de La Rioja, Obtenido de goo.gl/eLJLRe

Mihelcic, J., \& Zimmerman, J. (2012). Ingeniería ambiental. Fundamentos, sustentabilidad y diseño. Ciudad de México: Alfaomega.

Moreno, F. (2017). Ambiente y desarrollo sustentable: miradas diversas. Quilmes: Universidad Nacional de Quilmes.

Morin, E. (2011). La vía para el futuro de la humanidad. Buenos Aires: Paidós.

Naredo, J. M. (2010). Raíces económicas del deterioro ecológico y social. Más allá de los dogmas. Madrid: Siglo XXI.

Naredo, J. M. (2011). Reflexiones sobre la bandera del decrecimiento. Viento Sur, (118), 23-35.

Pindyck, R., \& Rubinfeld, D. (2018). Microeconomía. Madrid: Pearson Educación.

PNUD. (2016). Objetivos de desarrollo sostenible. Obtenido de Programa de las Naciones Unidas para el Desarrollo: goo.gl/nzXxbu

Polo, J. (2016). La teoría de la dependencia y la colonialidad del poder. Dos ángulos de una misma dominación. Revista San Gregorio, 11(1), 6-17.

Rapoport, M., \& Guiñazú, S. (2016). Raúl Prebisch: Historia, pensamiento y vigencia de la teoría de la transformación para el desarrollo de América Latina. Tiempo y Economía, 3(2), 55-77. http://dx.doi.org/10.21789/24222704.1129

Riechmann, J. (2004). Gente que no quiere viajar a Marte. Ensayos sobre ecología, ética y autolimitación. Madrid: Los Libros de la Catarata.

Rifkin, J. (2014). La sociedad de coste marginal cero. El Internet de las cosas, el procomún colaborativo y el eclipse del capitalismo. Barcelona: Paidós.

Salcedo, M., Rebolloso, F., \& Barber, C. (2010). El desarrollo sustentable. Modelo de conciliación entre el progreso económico, la justicia social y la preservación del medio ambiente. Gestión y Estrategia, (37), 17-31.

Sempere, J., \& Tello, E. (2007). El final de la era del petróleo barato. Barcelona: Icaria-CIP.

Sen, A. (2000). Desarrollo y libertad. Buenos Aires: Planeta.

SICES. (2018). Estadística Social. Obtenido de Sistema Integrado de Conocimiento y Estadística Social del Ecuador: goo.gl/gTcfmZ

Suárez, G. (2011). Crecimiento económico vs degradación ambiental: ¿Existe una curva de Kuznets ambiental en América Latina? Periodo 1970-2008. Quito: FLACSO-Ecuador.

Urquijo, M. (2014). La teoría de las capacidades en Amartya Sen. Revista Edetania, (46), 63 - 80.

Vergara, C., \& Ortiz, D. (2016). Desarrollo sostenible: enfoques desde las ciencias económicas. Apuntes Cenes, 35(62), 15-52. https://doi.org/10.19053/22565779.4240

WWF. (2016). Planeta Vivo. Informe 2016. Resumen. Obtenido de https://goo.gl/Xr1fcm

Zaar, M. (2018). Del decrecimiento al post-capitalismo. XV Coloquio Internacional de Geocrítica. Las ciencias sociales y la edificación de una sociedad post-capitalista (págs. 2-21). Barcelona: Universitat de Barcelona. 